\title{
Controlled release of copper from an intrauterine device using a biodegradable polymer
}

\author{
Reshmi Ramakrishnan, Bharaniraja B, Abi Santhosh Aprem*
}

HLL Lifecare Ltd, Corporate R \& D Centre, Trivandrum, Kerala, India-695017

${ }^{*}$ corresponding author

\section{Abstract}

Background: The adverse effects of copper intra uterine devices such as abnormal bleeding, pain and cramps may be due in part to the burst release of copper ions during the first few months of usage. This study focuses on controlling the initial burst release of copper ions.

Study design: This study evaluated in vitro release rates of copper for a period of one year from standard CuT380 intra uterine devices (IUD) (n=6)and from CuT380 IUDs coated with poly(DLlactide-co-glycolide) (PLGA) films $(n=6)$. This study characterized the coated device for its morphological changes during degradation of film by scanning electron microscopy (SEM).

Results: CuT380 IUDs coated with PLGA film with a thickness of $0.10 \pm 0.02 \mathrm{~mm}$ showed a reduced

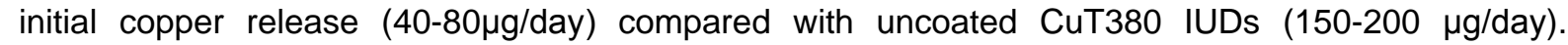
Statistically significant $(P<0.05)$ results were obtained at different time intervals during the overall study period of one year. SEM images showed degradation of coating.

Conclusion: Coating a CuT380 IUD with biodegradable polymer reduced the initial copper release without affecting release at one year. Clinical trials are required to determine whether this could reduce side effects such as bleeding and pain associated with copper containing intra uterine devices.

Keywords: Copper Intrauterine device, biodegradable polymer, PLGA, controlled release

\section{Introduction}

Copper T Intra uterine devices (IUD) represent an important contraceptive option for a very large number of women, about 160 million worldwide. The IUD, which is the most frequently used longacting reversible contraceptive method in the world, is used by 14 per cent of women of reproductive age [1]. A major drawback of copper IUDs is the high incidence of increased bleeding and pain

${ }^{*}$ Corresponding author. Tel.:0471-2774707; Fax : 0471-2774707

Email address:abi@lifecarehll.com (Abi Santhosh Aprem) 
following insertion. This may be related to a high initial release of copper [2-4]. It is also reported that $67 \%$ of women using CuT380 complained about menstrual side effects within the first year of use [5]. The corrosion rate of IUD is extremely high in the first few days after the insertion, which is the so called burst release of cupric ions [6 -8]. It is also reported that the high corrosion rates of the TCu380A IUD and TCu220C IUD in simulated uterine solution were obtained after the first and the third days of immersion in the solution respectively [9]. According to literature, burst release could contribute to abnormal bleeding $[2,10,11]$. Arancibia et al [12] demonstrated that a burst release of copper ions occurs in the first few months after insertion, and then the release rate decreases and stabilizes gradually. Hubacher et al [13] suggested that side effects like bleeding and pain decrease over time, which might mean that the burst release of copper is one of the causal factors.

Yang et al [14] studied the effects of low density poly ethylene (LDPE) film on the properties of copper/LDPE composites for intrauterine contraceptive device to reduce burst release of copper. Alvarez et al [15] also studied the effect of pre-treatment with organic inhibitors to reduce burst release of copper ions. In order to control the burst release of copper ions we designed a safe, biodegradable coating over the copper wire for controlling the release and achieving the recommended levels of copper for contraceptive effect which is reported to be in a range of 20 to 80 $\mu g /$ day [16].

Due to good biodegradability, biocompatibility and proper mechanical properties poly ( DL-lactide-coglycolide ) PLGA75/25, a biodegradable FDA approved polymer [17], was selected for this purpose. The degradation products are easily metabolized in the body and are eliminated as carbondioxide and water [18].

In this work, we engineered PLGA film and coated over the stem part of copper T to modulate the burst release of copper ions from the copper $\mathrm{T}$ device. The morphological changes during degradation of film coating over the Copper $\mathrm{T}$ were studied through scanning electron microscopy (SEM) analysis. The biodegradable polymer coating may prevent burst release, which in turn, may decrease side effects.

\section{Materials and Methods}

\subsection{Preparation of coated CuT380 IUD}

Preparation of CuT380 IUD coated with PLGA film (coated CuT380) involves preparation of PLGA film, coating of PLGA film onto CuT380 IUD (uncoated CuT380), packing and sterilization. Uncoated 
58 CuT380 is sourced from HLL Lifecare Ltd, India, who is a UNFPA prequalified manufacturer of Copper T.

Poly (D,L-lactide-co-glycolide) PLGA 75/25 (Corbion Biomaterials, Netherlands) was dissolved in ethylacetate (EA) under stirring. Solvents like tetrahydrofuran, methylene chloride, chloroform, ethyl acetate, hexafluoroisopropanol and acetone are found to be good solvents for the selected polymer PLGA. Based on the extent of solubility and its low toxic potential ethyl acetate was selected as the ideal candidate out of the mentioned solvents. As per ICH Q3C guidelines, Ethyl acetate solvent comes under class 3 solvents [19] which are regarded as less toxic and of lower risk to human health. Different $\mathrm{w} / \mathrm{v}$ concentration $(10 \%, 30 \%$ and $50 \%)$ of solutions were prepared and checked for the suitability of release and film formation ability with 3 different methods of coating such as dip coating, spray coating and film wrapping.10\%w/v PLGA solution was finalized for coating by film wrapping, after optimization.

The Polymer film (PLGA film) prepared with a thickness of $0.10 \pm 0.02 \mathrm{~mm}$ was sized into desired dimensions $(3 \mathrm{~cm} \times 1 \mathrm{~cm})$ and then was wrapped over the copper wire on the stem part of uncoated CuT380 to obtain the finished product coated CuT380 (Fig 1).

The finished product coated CuT380 was packed in Tyvek pouch as primary packaging and then subjected to sterilization by gamma radiation as per the standard ISO 11137 at a dose of $25 \mathrm{kGy}$.

\subsection{Characterization of coated CuT380}

The gamma sterilized coated CUT380 was characterized for its morphological changes during degradation of film by SEM, release of copper ions by UV.

\subsubsection{PLGA film Degradation}

The surface morphology of coated CuT380 samples was examined using Zeiss EVO 18 and HITACHI S2400 scanning electron microscope. The samples were sputter-coated with an $\mathrm{Au}-\mathrm{Pd}$ mixture to ensure conductivity. The coated CuT380 samples which were suspended and incubated in $50 \mathrm{ml}$ of simulated uterine solution (SUS) at a temperature of $37.0 \pm 0.1^{\circ} \mathrm{C}$ with $70 \mathrm{rpm}$. At predetermined time points, the samples were withdrawn and dried for $24 \mathrm{hrs}$ to investigate polymer film degradation by SEM. 
To compare the release behavior of coated CuT380 and uncoated CuT380, the release rate of copper was studied in the same environmental conditions. The coated CuT380 $(n=6)$ and uncoated CuT380 $(n=6)$ samples were suspended and incubated in $50 \mathrm{ml}$ of SUS a temperature of $37.0 \pm 0.1^{\circ} \mathrm{C}$ with 70 rpm.

The composition of SUS $(\mathrm{g} / \mathrm{L})$ is as follows.4.97 NaCl, $0.224 \mathrm{KCl}, 0.167 \mathrm{CaCl}_{2}, 0.25 \mathrm{NaHCO}_{3}, 0.50$ Glucose.0.072 $\mathrm{NaH}_{2} \mathrm{PO}_{4} .2 \mathrm{H}_{2} \mathrm{O}[4,8,20]$. The intra uterine $\mathrm{pH}$ is reported in the range from 6.0 to 7.9 [8]. Considering the above fact, tests were conducted in simulated uterine solution at $\mathrm{pH} 6.3$, which represents the condition with high copper corrosion rate [8].

Samples were taken after different exposure time (1,7 and 30 days initially and subsequently every month) and refreshed with SUS. The samples of incubated SUS solution were added to chelating agent (Diethyl ammonium salt of diethyl dithiocarbamic acid, with a concentration of $8 \times 10^{-3} \mathrm{~mol} / \mathrm{L}$ ) [20] and $\mathrm{Cu}^{2+}$ concentration was analysed by absorbance measurements using a UV Spectrophotometer (Model: Shimadzu 1700) with an absorption maximum at $448 \mathrm{~nm}$.

An unpaired, two tailed t-test was used to test the significance of difference in the release between coated CuT380 and uncoated CuT380. A p-value of 0.05 was defined to be statistically significant.

\section{Results:}

3.1. Characterization of coated CuT380

3.1.1. PLGA film degradation:

The erosion and degradation of PLGA film are considered as the factors influencing release of copper ions and are controlled by the physical properties of the film. Degradation of PLGA film occurs by bulk erosion [21]. The PLGA film started developing pores on it and the number of pores and its size increased over time leading to degradation of the film as observed in the micrograph pictures (Fig 2).

\subsubsection{Estimation of copper ions}

Fig 3 shows the release profile of coated CuT380 and uncoated CuT380 samples for 360 days. The initial release of copper (after $24 \mathrm{hrs)}$ ) from coated CuT380 is $40 \mu \mathrm{g} /$ day (mean $\pm \mathrm{SD}, \mathrm{n}=6$ ) and that of uncoated CuT380 is around $195 \mu \mathrm{g} /$ day $(\mathrm{n}=6)$. The release was statistically significant at a level of $p$ - 
value $<0.05$ when student unpaired t test was used. The results demonstrate that the initial release rate of copper ions in uncoated CuT380 was very high $(195 \mu \mathrm{g} /$ day $)$ and decreased gradually in 30

117 days $(165 \mu \mathrm{g} /$ day $)$ and maintain a constant release after 180 days $(40-80 \mu \mathrm{g} /$ day). The initial burst release of copper ions in uncoated CuT380 is thus clear, whereas, in coated CuT380 the release of copper ions was controlled at a rate of $40-80 \mu \mathrm{g} /$ day throughout the study period of 360 days.

\section{Discussion:}

121 The adverse effects of copper intra uterine devices such as abnormal bleeding, pain and cramps may be due in part to the burst release of copper ions during the first few months of usage. This study focuses on controlling this initial burst release of copper ions.

124 We developed here a coated CuT380 and demonstrated a better control of the burst release observed with the standard CUT380 IUD.

The coating material PLGA becomes more attractive as compared to natural hydrophilic polymer, because the release profile of the drug (copper ions) can be controlled by diffusion and the erosion of the polymer. Our results illustrate the release pattern of PLGA film on erosion.

In correlation with Arancibia et al [12] our research on in vitro copper release also demonstrates that the burst release of copper ions from uncoated CuT380 occurs only in the initial few months and then decreases and stabilizes gradually throughout the study period. The copper release study shows a controlled release of copper ions from coated CuT380 from the first day that is maintained $(40-80$ $\mu \mathrm{g} /$ day) throughout the study period of 360 days. Thus the drawback of the copper-bearing IUDs due to the initial burst release of copper ions (150-200 $\mu \mathrm{g} /$ day) in the initial days after insertion can be overcome by controlling the release rate as observed in coated CuT380.The initial cupric ion release from the coated CuT380 is significantly less than that of the existing uncoated CuT380 which may lead to less side effects related to burst release $[22,23]$. This needs to be clinically evaluated and will be reported later.

\section{Conclusion}

140 Copper corrosion from uncoated CuT380 after 24 hours is thrice than that of the coated CuT380.

141 These differences in the cupric ion release among the 2 IUDs decreases over time and after a period 142 of $4-5$ months both exhibit approximately equal release rate which is proved to be effective for contraception. In view of the above results, coated CuT380 may provide a new and improved 
reversible contraceptive method, which is expected to minimize the burst release of copper ions. We described an approach to suppress burst release and clinical testing is warranted.

\section{Acknowledgement}

The authors thankfully acknowledge the financial support received from Bill \& Melinda Gates Foundation under the Grand Challenge Explorations (\#OPP1025604) and HLL Lifecare Ltd, Trivandrum, Kerala, India.

\section{References}

[1] World contraceptive use, United Nations Population Division, 2009. Available from URL: http://www.un.org/esa/population/publications/contraceptive2009/contracept2009_wallchart_front .pdf

[2] Timonen H. Copper release from copper-T intrauterine devices. Contraception 1976;14(1):25-38.

[3] Zielske H, Koch U J, Badura R, LadeburgH.Studies on copper release from copper-T device (TCu200) and its influence on sperm migration in vitro. Contraception 1974;10:651-56.

[4] Jinying Li, Ying Li, XuanGu, YanliGao, JianpingLiu. Investigation of the release behavior of cupric ion for three types of Cu-IUDs and indomethacin for medicated Cu-IUD in simulated uterine fluid. Contraception 2008;77:299-302.

[5] Infection Prevention Practices for IUD insertion and Removal, By the United States Agency for $\begin{array}{lllll}\text { International } & \text { Development } & \text { (USAID), } & \text { Available } & \text { from }\end{array}$ :http://www.maqweb.org/iudtoolkit/service_delivery/infectionprevention.shtml

[6] Mora N, Cano E, Mora EM, Bastidas JM. Influence of pH and oxygen on copper corrosion in simulated uterine fluid. Biomaterials 2002;23: 667-71.

[7] Jie Gao, Ying Li, Jian-ping Liu, XuanGu.Releasing of cupric ion of three types of copper-bearing Intrauterine Contraceptive Devices in simulated uterine fluid. J Reprod Contra 2007;18:33-40.

[8] Zhang C, Xu N, Yang B.The corrosion behavior of copper in the simulated uterine fluid. CorrosSci 1996;38:635-41.

[9] Bianmei Cao, Tingfei Xi and YudongZheng.Releasebehavior of cupric ions for TCu380A and TCu220 IUDs. Biomed Mater 2008;3:044114.

[10] Xu T, Lei H, Cai SZ, Xia XP, Xie CS. The release of cupric ion in simulated uterine: Material nano-Cu/low-density polyethylene used for intrauterine devices. Contraception 2004;70:153-57. 
[11] Shuizhou Cai, Xianping Xia, Changsheng Xie. Corrosion behavior of copper/LDPE nanocomposites in simulated uterine solution. Biomaterials 2005;26:2671-6.

[12] Arancibia V, Pena C, Allen HE, Lagos G. Characterization of copper in uterine fluids of patients who use the copper T-380A intrauterine device. ClinChimActa 2003;332:69-78.

[13] David Hubacher, Pai-Lien Chen, Sola Park.Side effects from the copper IUD: do they decrease over time? Contraception.2009;79(5):356-62.

[14] Zhihong Yang, Changsheng Xie, ShuizhouCai, XianpingXia.Effects of LDPE film on the

[23] Zhang C, Xu N, Yang B.The corrosion behavior of copper in simulated uterine fluid. Corrosion 
207 Fig.1. Images of coated CuT380 and uncoated CUT380

208

Fig.2. SEM micrographs of PLGA film on coated CuT380 kept in SUS for (a) 1day, (b) 20 days

209

(c) 40 days

210 Fig.3. Release of copper from coated CuT380 and uncoated CuT380 (mean \pm SD, n=6)

211 




$H V: 10 \mathrm{kV}$

Mag: $2000 x$

SCTIMST

WD: $10 \mathrm{~mm}$

$10 \mu \mathrm{m}$ .

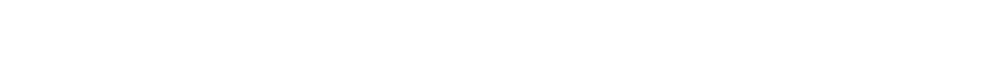

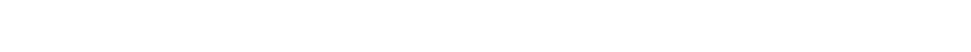
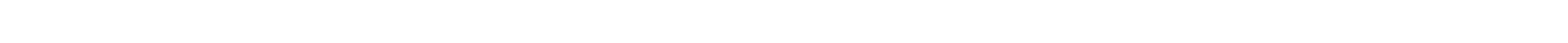

.

$$
\text { . }
$$




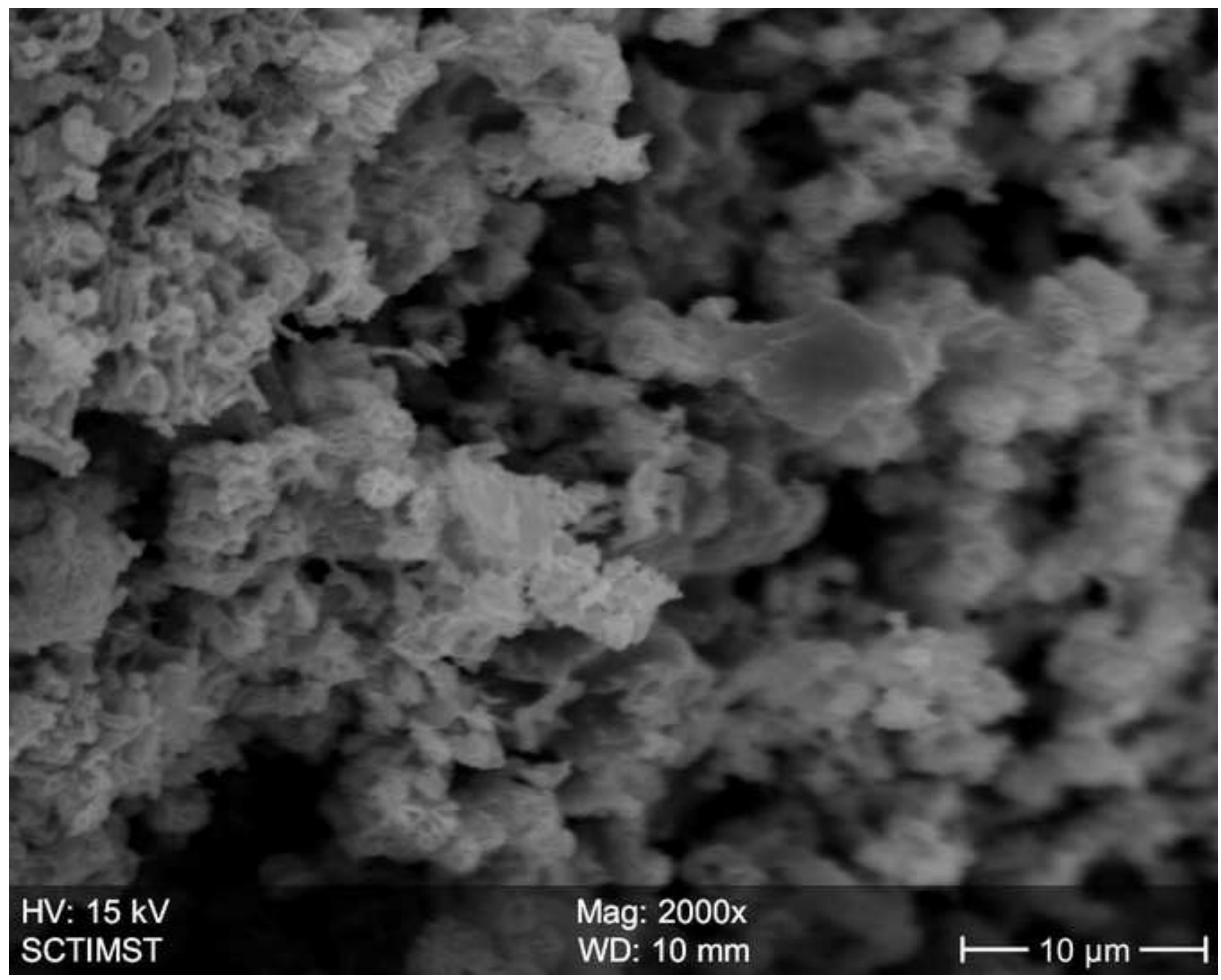

HV: $15 \mathrm{kV}$

Mag: 2000x

WD: $10 \mathrm{~mm}$

$10 \mu \mathrm{m}$

, 


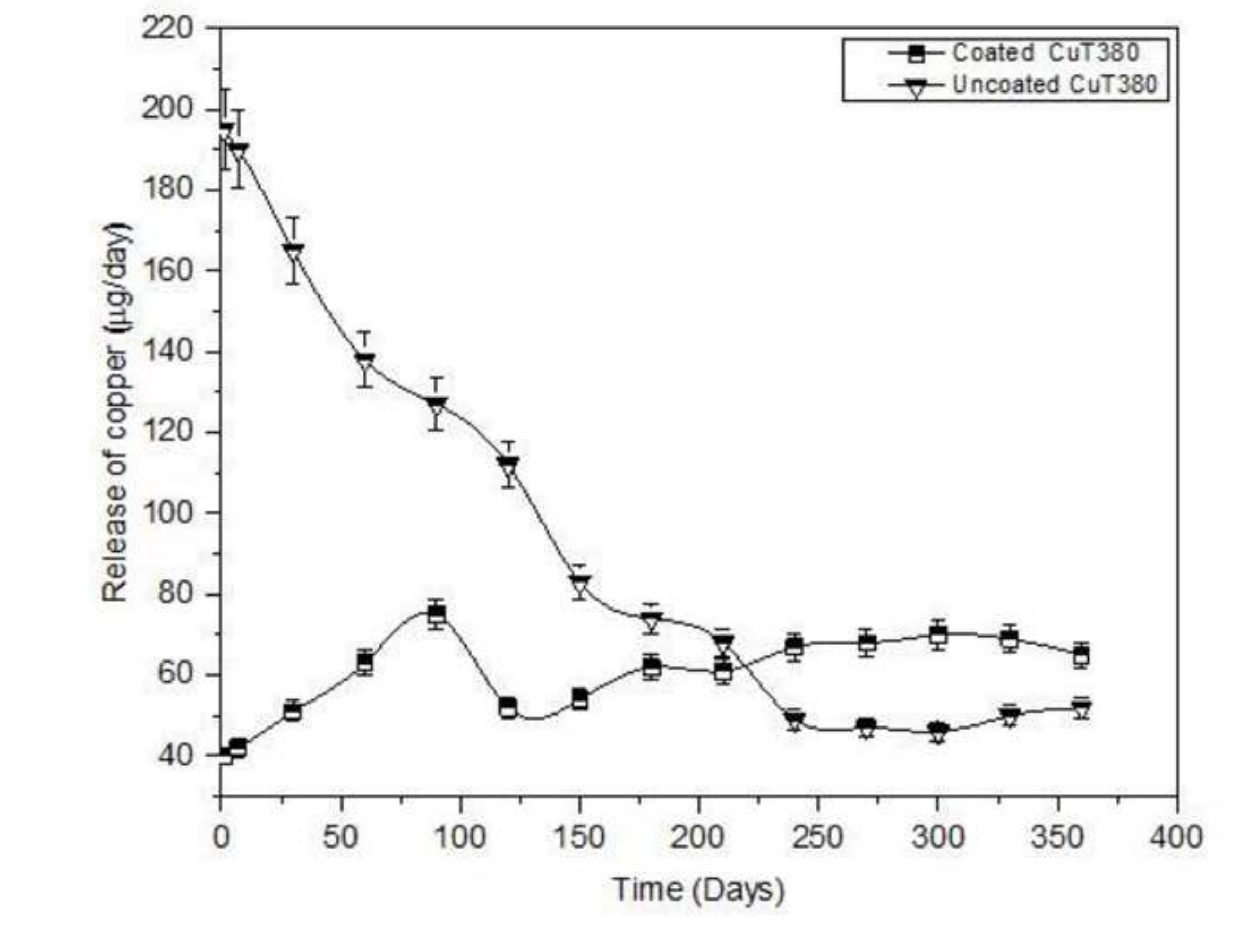

Time (Days)

更

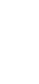

.

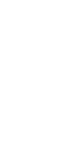

